\title{
Integrated Computational Materials Engineering in Solar Plants: The Virtual Materials Design Project
}

\author{
FRANCISCO MONTERO-CHACÓN ${ }^{1,6},{ }^{1,6}$ MICHELE CHIUMENTI, ${ }^{2}$ \\ JAVIER SEGURADO, ${ }^{3,4}$ and MANUEL DOBLARÉ ${ }^{5}$
}

\begin{abstract}
1.-Dpto. Ingeniería, Universidad Loyola Andalucía, Calle Energía Solar 1, 41014 Seville, Spain. 2.-International Center for Numerical Methods in Engineering (CIMNE), Technical University of Catalonia (UPC), Edificio C1, Campus Norte, Gran Capitán s/n, 08034 Barcelona, Spain. 3.-IMDEA Materials Institute, C/Eric Kandel 2, 28906 Getafe, Madrid, Spain. 4.-Department of Materials Science, Polytechnic University of Madrid, 28040 Madrid, Spain. 5.-Aragon Institute of Engineering Research (I3A), University of Zaragoza, C/Mariano Esquillor s/n, 50018 Saragossa, Spain. 6.—e-mail: fpmontero@uloyola.es
\end{abstract}

The high temperatures required for efficient operation of solar thermal power plants constitutes one of the major challenges of this technology. Gaining insight into materials behavior at very high temperatures is critical to improve their techno-economic feasibility. Standard material characterization approaches become inefficient, as extensive testing campaigns are required. We propose a multiscale-multiphysical approach that accounts for materials composition to (1) predict the behavior of both Inconel 625 and new solar salts, and (2) assess the thermomechanical performance of key components. We carried out a complete thermoelastic multiscale analysis that spans six time and length scales in a single simulation platform, combining discrete and continuum tools (from quantum to continuum mechanics). These applications show the substantial economic benefits that may be achieved by an ICME approach in the energy sector, reducing the cost of prototypes while decreasing development times and maintenance costs due to a better understanding of materials behavior.

\section{INTRODUCTION}

Solar energy has become an interesting sustainable alternative to fossil fuel combustion, experiencing an essential impulse in the last years. ${ }^{1}$ Moreover, solar energy plants have proven to be the best choice at specific locations, especially in remote areas. In this sense, the Atacama-1 project, a hybrid photovoltaic (PV) and concentrated solar power (CSP) plant (Atacama Desert, Chile), was claimed to be the first commercial plant in which this type of energy was the most efficient solution both economically and environmentally. ${ }^{2}$ However, the global market penetration of these type of plants is still restricted by their current high levelized cost of energy, compared to traditional oil-based energy production. This problem can be tackled by increasing the cycle efficiency and designing high-capacity energy storage systems. ${ }^{3}$ Both cases require an increase of the operating temperature. However, such an increase may cause side effects in the materials performance, sometimes leading to the failure of critical components (e.g., receivers, heat exchangers), among other significant implications.

Materials characterization in key CSP plant components at these higher temperatures is therefore of great importance in the assessment of their technical and economic feasibility. The use of classical phenomenological constitutive models becomes very inefficient under this scenario, as it requires testing materials at many different conditions. Moreover, changes in material microstructure and operation conditions would imply repeating the characterization procedure and adjustment of several constitutive models.

An alternative approach, based on multiphysical and multiscale analyses, is undoubtedly ideal because it would provide the virtual material response as a function of the material composition, microstructure, and working conditions. This type 
of approach would also reduce the number of tests, resulting in a faster evaluation of the suitability of a particular material in a specific application. Under this paradigm, the analysis of different physical phenomena involved at different time and length scales of observation (i.e., an atoms-to-components strategy) is required. Thus, the use of numerical techniques enables the design process. It is for this reason that we followed an Integrated Computational Materials Engineering (ICME) approach ${ }^{4}$ to gain insight into the several physical phenomena involved in solar energy harvesting and storage. Namely, for the characterization of the substrate material used in the receiver tube (in this application, Inconel 625 or IN625) and the performance of new thermal energy storage (TES) systems.

The idea of using a multiscale scheme for the development of virtual materials testing tools was first proposed in the MURI 99 project, ${ }^{5}$ which was accomplished by transferring the information obtained at one scale to the next one, from the electronic to the macrostructural level. The National Nuclear Security Administration launched in 2008 the "Predictive Science Academic Alliance Program", whose primary goal was to reduce the number of nuclear experiments by relying on numerical simulations. ${ }^{6}$ This can be seen as the origin of the ICME approach, whose objective is to combine the expertise achieved at different scales of observation to enhance the engineering design process.

The ICME methodology has been rapidly adopted by the industry, primarily by the aerospace and automotive sectors, ${ }^{7}$ allowing for an important reduction of the number of large-scale experiments. ${ }^{8}$ In the first case, the strict regulations on structural components certification still require a large number of tests. Nevertheless, this certification process can be accelerated within the ICME paradigm, as shown in Refs. 9 and 10. Moreover, this method has been successfully used in airframe ${ }^{11-13}$ and turbine design. ${ }^{14,15}$ A summary showing the maturity level of the ICME methodology in aeronautics can be found in Ref. 16.

Regarding the automotive industry, ICME has been used to analyze new manufacturing processes, ${ }^{17}$ the long-term performance of components, ${ }^{8}$ or in the design of a new generation of materials. ${ }^{18-20}$ In this sense, vehicle efficiency and product optimization have been the main objectives. ${ }^{21,22}$

As outlined in Ref. 8, the ICME approach can also be applied to the energy sector to enable greener, safer, and more sustainable energy technologies. $^{23,24}$ However, to our knowledge, the application of ICME in the energy sector has not yet been sufficiently covered in the literature.

In this work, we present an ICME-based approach for the analysis and design of key components in solar plants. For this purpose, we follow a hierarchical upscaling strategy to predict the materials behavior involved in energy harvesting and storage. In the case of the harvesting application, we present an analysis of IN625 superalloy solar receiver tubes. Although similar polycrystalline Nibased alloys have already been studied, ${ }^{25}$ their study was limited to a few scales of observation (typically, close to the microscale). In our work, however, we present a full multiscale thermoelastic characterization of IN625 tubes, from the electronic to the macroscopic scale. In addition, the non-linear behavior of this superalloy has been studied here at specific scales to understand certain physical phenomena (e.g., grain boundary sliding), which are related to long-term issues such as creep or thermal fatigue. Regarding the energy storage application, we present an analysis on the thermomechanical performance of new designs of TES systems, by following the same ICME strategy, including the thermophysical characterization of new phasechange materials (PCM) used in these devices.

This work was carried out under "The Virtual Materials Design Platform" project, led by Abengoa Research, the corporate research center of the international company Abengoa. As a result, an in-house simulation platform was developed for the analysis of relevant multiscale thermomechanical problems, combining discrete and continuum-based tools.

In "The Virtual Materials Design (VMD) Project: From Atoms to Solar Plants" section, the scales of observation subjected to study are presented according to the different physical phenomena involved. Relevant details on the working methodology followed throughout the project are provided. "Multiscale Multiphysical Assessment of Solar Energy Harvesting and Storage Components" section shows the integration strategy of the scales within a hierarchical methodology. For this purpose, two reference problems, namely, the thermomechanical analysis of the receiver tubes and the design of TES systems are presented. Finally, "Software" section describes the different modules of the platform and the workflow designed within the Virtual Materials Design (VMD) Project.

\section{THE VIRTUAL MATERIALS DESIGN (VMD) PROJECT: FROM ATOMS TO SOLAR PLANTS*}

The primary objective of the VMD project, which was first introduced in Abengoa Research's Strategic Agenda, ${ }^{26}$ was the development of a multipurpose simulation platform to predict the behavior of complex materials in renewable energy applications at different scales of observation. Therefore, the macroscopic behavior of novel materials is derived from the lowest scale of observation and regardless of any input parameters, following a pure in silico form. Although several problems of interest were explored within the VMD project, ${ }^{26}$ the platform

\footnotetext{
*The title of this section is inspired by Horstemeyer's book, ${ }^{8}$ Chapter 7: "Case study: from atoms to autos: a redesign of a Cadillac control arm".
} 
was built on two reference applications, namely, the virtual testing and characterization of (a) Inconel 625 solar receiver tubes and (b) PCM-based TES systems.

\section{Scales of Observation}

The major conceptual challenge in this project is to not only perform analyses at specific length and time scales but also establishing links between them. Indeed, the physical phenomena under consideration cover, at least, ten orders of magnitude throughout the time and length scales, from femtoseconds to seconds (or even hours or days in the case of creep or fatigue) and from ångströms to meters, respectively (Fig. 1). Therefore, a multiscale multiphysical approach is mandatory in order to understand the materials behavior at high temperature. A total of six scales of observation were considered in this study and are detailed next:

- Electronic $(\AA, \mathrm{fs})$ : at this scale of observation, quantum mechanics describes the interaction between the different atoms (and their corresponding subatomic particles such as electrons) in a specific system. As described in the next section, we used the density functional theory (DFT) to analyze the electronic dynamics ab initio (i.e., using physical constants only). This strategy allows linking the material composition and its behavior.

- Atomic (nm, ps): interesting material features emerge at this scale regarding the behavior of single phases (e.g., crystals) and interfaces (e.g., grain boundaries). Therefore, atomistic modeling techniques such as molecular dynamics (MD) and molecular statics (MS) are used to determine relevant material information such as the stacking fault energies, thermo-elastic behavior, or dislocation mobility of the IN625 crystals, for instance.

- Sub-microscopic ( $\mu \mathrm{m}, \mathrm{ns})$ : in some cases, the atomistic models mentioned above cannot appropriately address certain phenomena involved at higher time scales, such as the grain boundary kinetics. In this work, a kinetic Monte Carlo (KMC) model is used to tackle this problem. In addition, important thermal properties (e.g., phase diagrams, specific heat) can be identified at this scale, especially for the different species considered in the study (i.e., those of IN625 and new solar salt blends). This identification is carried out here using computational thermodynamics (CTD) simulations.

- Microscopic $(\mu \mathrm{m}, \mu \mathrm{s})$ : the material structure fulfills the continuum mechanics premise at this scale. Thus, continuum-based tools such as the finite element method (FEM) and the fast Fourier transform (FFT) are used to characterize IN625 polycrystals subjected to different temperature conditions, which provide relevant information for the constitutive models used in simulations at higher scales.

- Mesoscopic (mm, ms): in this project, the mesoscale lays between the micro- and macroscales, but from the continuum mechanics side. Interesting problems related to the material integrity of components can be analyzed at this scale through unit cell or local FEM simulations.

- Macroscopic (m, s): the feasibility of the solar plant systems (i.e., receiver and heat exchanger) is subject of study at this scale. Typically, coupled thermomechanical full-scale FEM simulations are carried out, whose input material properties are obtained with the previous lower scale simulations.

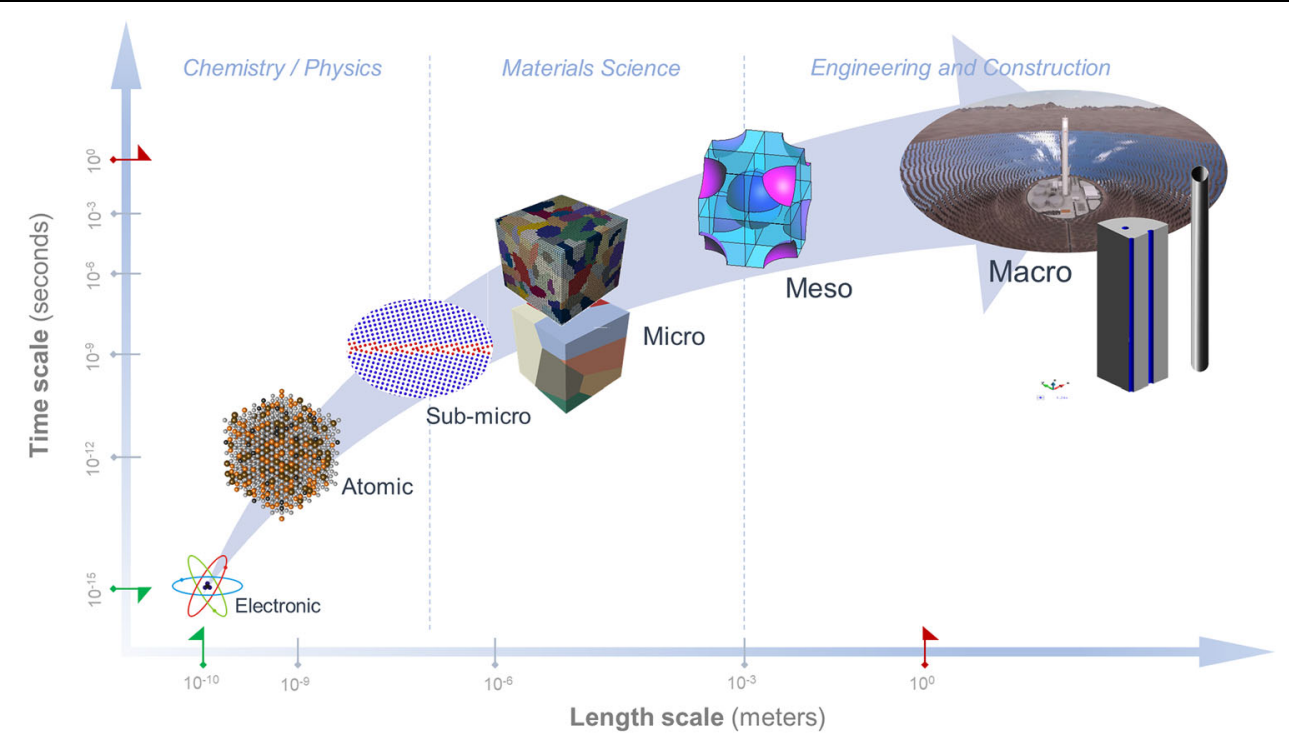

Fig. 1. From atoms to solar plants: length and time scales of observation and disciplines involved in the VMD project. 
As discussed in the next section, the central multiscale scheme adopted in this project is the hierarchical upscaling technique. Thus, unidirectional handshakes are performed at the boundaries of the scales described above, employing informationpassing techniques. ${ }^{27}$ Other strategies such as nested communication (i.e., $\left.\mathrm{FE}^{2}\right)^{28}$ or concurrent multiscale methods ${ }^{29}$ were also implemented in our simulation platform. ${ }^{30}$

\section{The VMD Platform Project}

The development of a tailor-made ICME platform for the analysis and design of components for solar plants is not only a challenge from the technical point of view but also from the management one. It involves the resolution of scientific problems, software integration, and the interaction of cross-disciplinary working groups. For the sake of simplicity, we adopted a problem-type approach. Therefore, several reference problems present in solar plants owned by Abengoa were analyzed, paying particular attention to the thermomechanical performance of structural and functional materials.

Within the scope of the VMD project, the achieved milestones were the identification of state-of-the-art simulation platforms, the identification of the most suitable simulation tools, and the development of two modules: one for MD simulations and another for coupled thermomechanical FEM simulations. Hence, different work groups were in charge of the analysis of these problems at specific scales of observation.

The VMD project was led by Abengoa Research with the collaboration of specialized research groups, namely, IMDEA Materiales (Madrid, Spain), the International Center for Numerical Methods in Engineering CIMNE (Barcelona, Spain), and the Nanostructured Materials for Technological Applications research group at the University Pablo de Olavide (Sevilla, Spain). This project involved more than 30 researchers, from 2012 to 2016. As a result, the technology readiness level (TRL) of the platform increased from TRL 2 to 5 , which in terms of software technology implies passing from the formulation of the concept to the software validation in a relevant environment.

\section{MULTISCALE MULTIPHYSICAL ASSESS- MENT OF SOLAR ENERGY HARVESTING AND STORAGE COMPONENTS}

In this section, the ICME methodology adopted within the VMD project is presented from two applications: (1) the thermomechanical characterization of IN625 solar receiver tubes, and (2) the thermomechanical assessment of a new PCM-based TES system. In both cases, a hierarchical upscaling technique was used to obtain relevant material parameters that were subsequently passed from one level to a higher one.

\section{Thermomechanical Characterization of IN625 Solar Receiver Tubes}

Solar receiver tubes receive the sunlight from myriads of mirrors on the solar field and are responsible for heating the so-called heat transfer fluid, typically a synthetic oil or molten salt, and should absorb as much solar radiation as possible. Solar absorption can be enhanced with the use of socalled solar selective coatings (SSCs), which are a class of multi-layered cover deposited onto the substrate material to increase the absorptance and decrease the emissivity of the assembly. The structural stability of SSC layers at high temperature has already been studied in Ref. 30. In the present work, we focus on the behavior of the substrate material, IN625, at high temperature. Different phenomena, such as the settlement of thermal and mechanical properties (and their dependency on the temperature), the behavior of grain boundaries or the effect of thermal cycling, are explored in this work following a bottom-up upscaling strategy.

\section{Development of Potentials for Ni-Based Alloys}

The electronic scale is the most basic level at which matter can be currently studied. Ab initio methods, which only depend on physical constants, were used to determine relevant atomic information between the different elements of the compounds (e.g., potentials, activation energy, free energy). DFT simulations were used to evaluate the interaction between $\mathrm{Ni}, \mathrm{Cr}, \mathrm{Mo}$, and $\mathrm{Fe}$, which are the main constituents of IN625. It must be remarked that this superalloy barely presents precipitates; therefore, it can be considered as a single FCC phase presenting its elements in solid solution. The electronic study considering all these elements goes beyond the characterization of IN625 and supposes a first step towards developing an effective molecular potential valid for different compositions that would allow investigating the effect of the particular composition in the thermoelastic behavior.

As a first attempt, and due to the lack of appropriate interatomic potentials for $\mathrm{Ni}-\mathrm{Cr}$ alloys in the literature, a Johnson-type embedded atom method (EAM) potential was optimized based on experimental observables (e.g., lattice parameter, cohesive energy, elastic constants, or the coefficient of thermal expansion). Thus, DFT simulations were carried out to validate the parameterized potentials by checking the lattice parameter and cohesive energy. In such a way, an ab initio route was found for the definition of the potentials, as detailed in Ref. 31. The accuracy of this potential was proven successful for predicting the behavior of $\mathrm{Ni}-\mathrm{Cr}$ compounds; nevertheless, the same process failed when third species such as Mo or Fe were taken into account. For this reason, an automatic parameterization of the potentials, purely based on DFT simulations, were successfully used for fitting the electron density and the short-range terms of the 
EAM potentials for Ni-Cr-Mo-Fe alloys, with no use of experimental data. ${ }^{32}$ This first step is shown in Fig. 2a. The development of the potential is detailed in Ref. 32.

From the thermodynamic point of view, DFT simulations were also used to predict the miscibility gap (Fig. 2a), which is a requirement in the CTD simulations of the phase equilibria of the Ni-Cr-MoFe system. The analysis of the Cr-Mo subsystem is presented in Ref. 33.

\section{Characterization of Crystals and Grain Boundaries}

The MD potentials developed in "Development of Potentials for Ni-Based Alloys" section were implemented in atomistic simulations to characterize the behavior of single phases (i.e., IN625 crystals) and their interfaces (i.e., grain boundaries). Moreover, the atomistic modeling scope was broader in the VMD project; in fact, other phases such as bulk amorphous carbon and $\mathrm{TiC}$ nanoparticles, present in the SSC's absorber layer, were analyzed with other existing potentials (e.g., REBO, AIREBO, 2NN-MEAM). ${ }^{30}$

Atomistic models (i.e., MD and MS) were used to obtain the stacking fault energies, thermoelastic behavior, and dislocation mobility of crystals, among other properties. In particular, MD simulations of an IN625 crystal with a few thousand atoms (Fig. 2b) were carried out to evaluate the density, elastic constants (C11, C12, and C44), and coefficient of thermal expansion (CTE), in a temperature range up to $1200 \mathrm{~K}$. An in-depth analysis of this characterization is reported in Ref. 32. As observed in Fig. 2b, the agreement of the computed CTE with that of the ASTM standard ${ }^{34}$ is remarkable, even though no experimental input was used. Moreover, the platform allows the determination of thermophysical properties such as the thermal conductivity $(K)$ or specific heat $\left(C_{\mathrm{p}}\right)$, as seen for the SSCs phases. ${ }^{30}$

In addition, simulations considering two grains made of the principal constituent were also performed to determine the grain boundary (GB) mobility and interaction with dislocations (Fig. 2b). In fact, it is well known that GB plays a fundamental role in creep life at high temperatures through grain boundary sliding and the diffusion of vacancies (from and through the GB), and are also responsible for intergranular cracking in static and fatigue conditions. ${ }^{35-37}$ Thus, MD simulations of a bicrystal with specific crystallographic orientations were made using a simple $\mathrm{Ni}$ potential to alleviate the complexity arising from using the complex multi-component potential. This MD study provided the energy, stress-strain response, and GB displacement of the interface at different temperatures up to $900 \mathrm{~K}$ (see Fig. 2b).

Although corresponding to a larger scale of observation (i.e., the sub-microscale), a KMC model was also developed to account for the atomistic simulations (i.e., molecular statics and nudged elastic band) to extract the minimum energy path. ${ }^{38}$ The resulting model allowed the simulation of GB sliding and coupled migration at a higher time scale, reaching deformation rates more than four orders of magnitude larger than that of MD (Fig. 2b), but still in excellent agreement with the MD simulations. The information provided by this model can be used to define the shear-sliding cohesive laws in the polycrystal FEM model described in "Thermomechanical Behavior of IN625" section.

Another aspect studied with MD was the interface behavior of amorphous carbon matrices reinforced with $\mathrm{TiC}$ nanoparticles, namely, the debonding mechanism leading to a decrease in the thermomechanical performance of SSC. ${ }^{30}$

The information obtained with the atomistic simulations were upscaled in terms of single-phase temperature-dependent mechanical properties (e.g., $\mathrm{C} 11, \mathrm{C} 12, \mathrm{C} 44$, or CTE) and interfacial tractionseparation laws, available for microstructural simulations.

\section{Thermomechanical Behavior of IN625}

The material behavior of IN625 polycrystals was characterized using mean-field methods. ${ }^{39-41}$ For this purpose, the single crystal thermal-dependent properties obtained in "Characterization of Crystals and Grain Boundaries" section were used. Random crystal texture and different temperatures were considered. ${ }^{32}$ The results for the elastic modulus and Poisson's ratio are plotted in Fig. 2c. It can be seen that the adjustment is reasonable, taking into account that no experimental data were used. In addition, an FFT-based homogenization analysis of a polycrystal containing ca. 2000 grains (Fig. 2c) was carried out in order to determine the elastic constants at different temperatures, reaching very similar values to those provided by the analytical method (see Ref. 32 for more details).

Regarding the inelastic behavior of IN625 polycrystals, different strategies were tested within the VMD project. First, classical plastic-hardening FEM simulations were carried out for polycrystals with given textures (Fig. 2c). Then, a crystal plasticity finite element method (CPFEM) model ${ }^{29}$ was tested for the analysis of the local behavior of a dozen grains (Fig. 2c). However, as pointed out above, the polycrystal behavior at high temperature is governed by GB effects. For this reason, cohesive elements were then implemented in the platform to model the interface behavior (Fig. 2c), as suggested in Ref. 42. However, the combination of both models is still under development and limited to a low number of grains. For the cyclic behavior, an equivalent FEM micromechanical thermal damage model, defined in terms of damage initiation and evolution rules, was also successfully tested. 


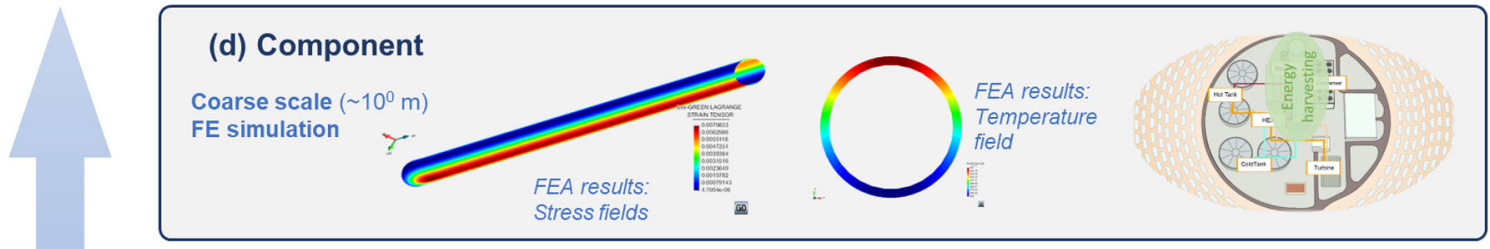

\section{(c) Material}

Microstructure ( $10^{3}$ grains)

FFT-based homogenization

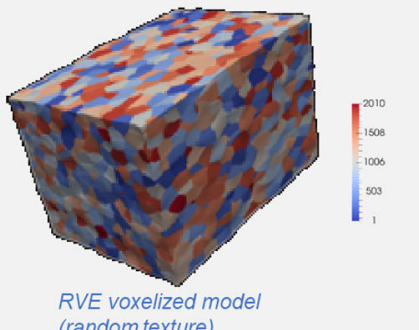

(random texture)
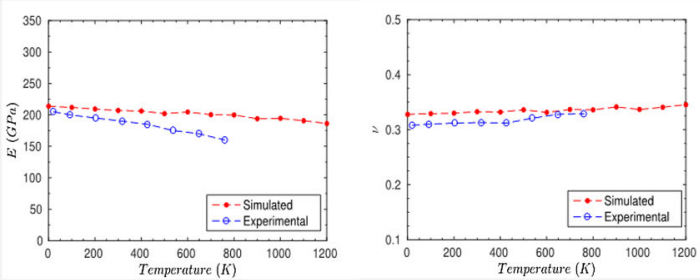

Elastic modulus and Poisson's ratio

Microstructure ( $10^{1}$ grains)

FE simulation

CPFEM RVE analysis
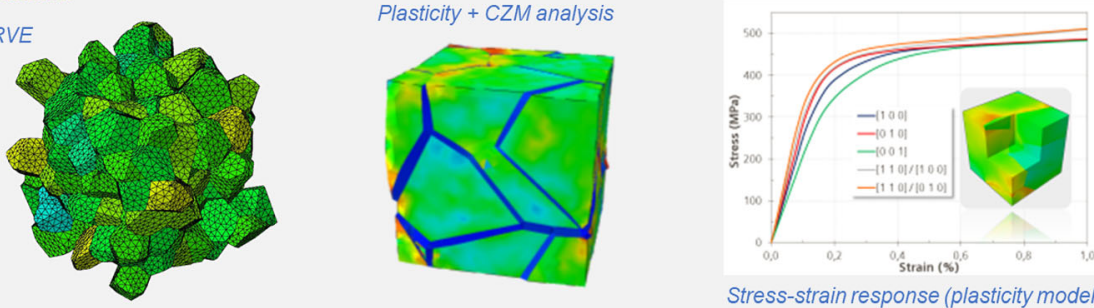

Stress-strain response (plasticity model)

\section{(b) Crystals and GBs}

Crystal ( $10^{3}$ atoms) MD simulation

\begin{tabular}{|c|}
\hline $62 \% \mathrm{Ni}$ \\
$23 \% \mathrm{Cr}$ \\
$10 \% \mathrm{Mo}$ \\
$5 \% \mathrm{Fe}$ \\
\hline
\end{tabular}

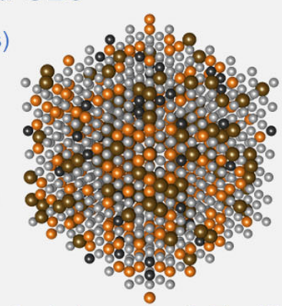

Thermomechanical response: $C_{j i}(T), \alpha(T)$

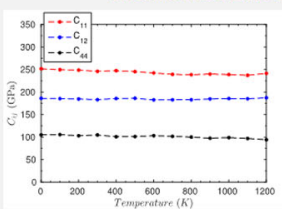

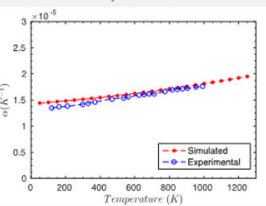

Grain boundary MD \& KMC simulations

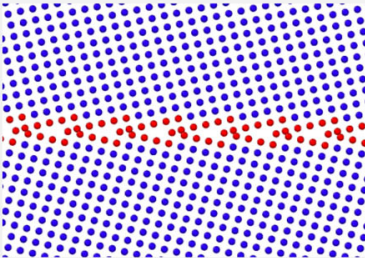

Shear stress-strain ( $T=800 \mathrm{~K})$

Average stress $(T)$
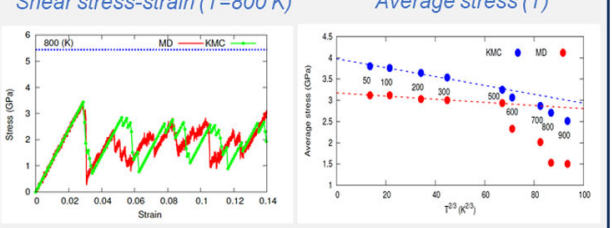

(a) Potentials

DFT simulations (Ni, Cr, Mo, Fe)

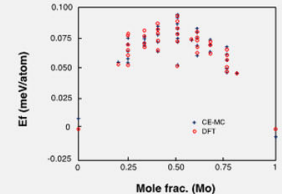

Energy of formatio

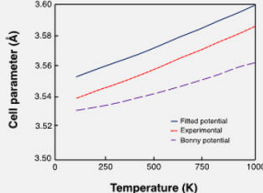

Cell parameter (fitting)

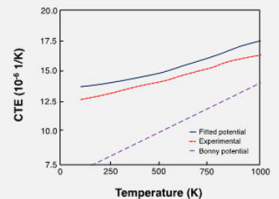

Thermal expansion (fitting)

Fig. 2. Bottom-up multiscale analysis of IN625 solar receiver tubes: (a) potential development, (b) determination of elastic constants and CTE of single grains and GB sliding characterization (reprinted with permission from Ref. 38), (c) FEM and FFT simulations of polycrystals, and (d) thermomechanical FEA of a receiver tube. 
One important aspect of microstructural modeling is microstructure generation. Hence, representative volume elements (RVE) of polycrystals were generated using Voronoi tessellations, including their grain texture.

Microscale simulations play a fundamental role in the VMD platform as they can be used to provide single-scale results. Combined with meso- and macroscale tools, typically FEA software, they can assess the thermomechanical performance of the tubes (Fig. 2d). This link can be arranged in the platform in a sequential form, ${ }^{27}$ or a concurrent multiscale scheme, ${ }^{28,29}$ to evaluate the constitutive tensor of the macroscale problem, if necessary.

Finally, we must remark that receiver tubes usually experience substantial temperature gradients, which in some cases exceed $100 \mathrm{~K}$ in a given section. This temperature field can also be computed from higher-resolution models that include the different heat transfer mechanisms present in the receiver cavity (convection plays an important role here).

\section{Thermomechanical Characterization of New PCM-Based TES Systems}

The main goal regarding the energy storage application considered is the characterization of new PCM-based TES systems. TES blocks are of great importance in CSP plants, as they are responsible for storing thermal energy (usually in the form of sensible heat) and provide electricity when solar radiation is not available (e.g., during the night). The economic feasibility of CSP plants largely depends on the thermomechanical performance of the TES block. Therefore, the technical assessment of TES systems is relevant. In this application, we present a heat exchanger (Fig. 3a) composed of thousands of PCM capsules arranged within a highconductivity matrix (Fig. 3b). Of course, the identification and characterization of new PCM materials are of great interest and, in this case, a new type of solar salt, which is a nitrates blend $(60 \%$ NaNO3 and $40 \%$ KNO3) enhanced with third species (e.g., $\mathrm{Li}, \mathrm{Cs}$ ), was tested. In this case, we adopted a topdown multiscale strategy for the analysis of the heat exchanger at three levels: system, component, and material. The application presented here is for demonstration purposes (i.e., confidential information has been omitted).

\section{System Level}

The system level belongs to the macroscale and, in this application, corresponds to the analysis of the heat exchanger (HEX), tens of meters long. Thus, we analyze a specific device with the use of coupled thermomechanical FEA, accounting for the heating and cooling rates provided by the solar field. These simulations allowed the identification of the thermal stresses along this device (Fig. 3a). The thermophysical properties were obtained by means of a refined analysis, namely the simulation of a cross-section of the HEX (Fig. 3b). Thus, a local macroscale analysis was carried out first considering the heterogeneity of the system by accounting for thousands of PCM capsules and the matrix, and the time evolution of the temperature field was obtained (Fig. 3b). Next, an equivalent homogenized model, including the phase-change process, was developed so as to compute the thermal stresses within that section (Fig. 3c). Another important parameter analyzed with this model is the global thermal inertia of the HEX. The homogenized model also reduces the computer times in a significant manner.

\section{Component Level}

As shown by the macroscale simulations, some critical spots were identified close to the distribution pipes. Thus, a mesoscale thermomechanical FEA at the component level (i.e., a cell containing tens of PCM capsules with a few millimeters of diameter) was carried out to identify the phase-change process and determine the thermal stresses.

A periodic unit cell analysis of the component (i.e., a cell containing one capsule with its corresponding PCM material and surrounding matrix, as shown in Fig. 3b) was also analyzed with the FEM to characterize the local thermal inertia. Thus, the solidification and melting times were computed, and the thermal stresses, both in the capsule and matrix, were obtained and compared to their corresponding limits. Thermal cycling promotes the apparition of local thermal stresses that deteriorate the long-term performance of the system.

\section{Computational Thermodynamics: Design of New Solar Salts}

Finally, at the material level, computational thermodynamics (CTD), precisely, the CALPHAD method, ${ }^{43}$ was used to determine the thermophysical properties of new blends of solar salts. ${ }^{44}$ This method was also used to characterize the Inconel alloy, as discussed in Ref. 33.

The CALPHAD method was adopted to quantify the effect of third components (e.g., $\mathrm{LiNO}_{3}, \mathrm{CsNO}_{3}$ ) when added to ordinary solar salt (i.e., $\mathrm{NaNO}_{3}$ and $\mathrm{KNO}_{3}$ ), especially on the resulting phase diagrams and the specific heat, which is responsible for the sensible storage capacity of the device.

Another aspect that was analyzed with CALPHAD was the latent heat capacity of the mix (i.e., fusion enthalpy, $\Delta H_{\mathrm{f}}$ ), which can be combined with the sensible mechanism so as to increase the total thermal storage capacity. These results are not only useful at the current scale to identify which 


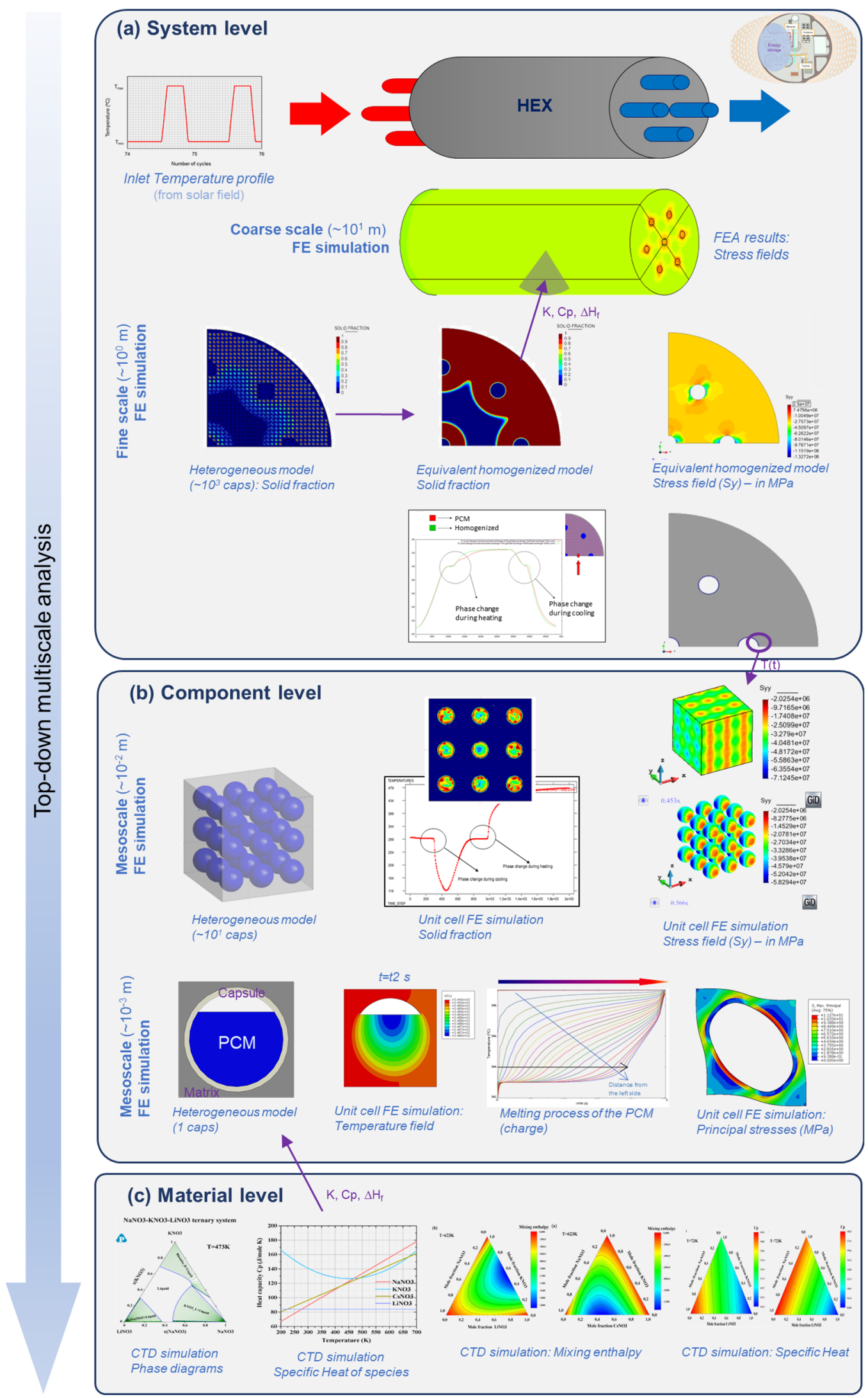

Fig. 3. Top-down multiscale analysis of PCM-based TES systems: (a) macroscale thermomechanical FEA of the HEX and refined simulations of a cross-section including its equivalent homogenized model, (b) component level analyses considering multiple and single capsule unit cells during the heating and cooling process, and (c) CTD-driven material level analyses of new solar salts considering their phase-change behavior (reprinted with permission from Ref. 47). 


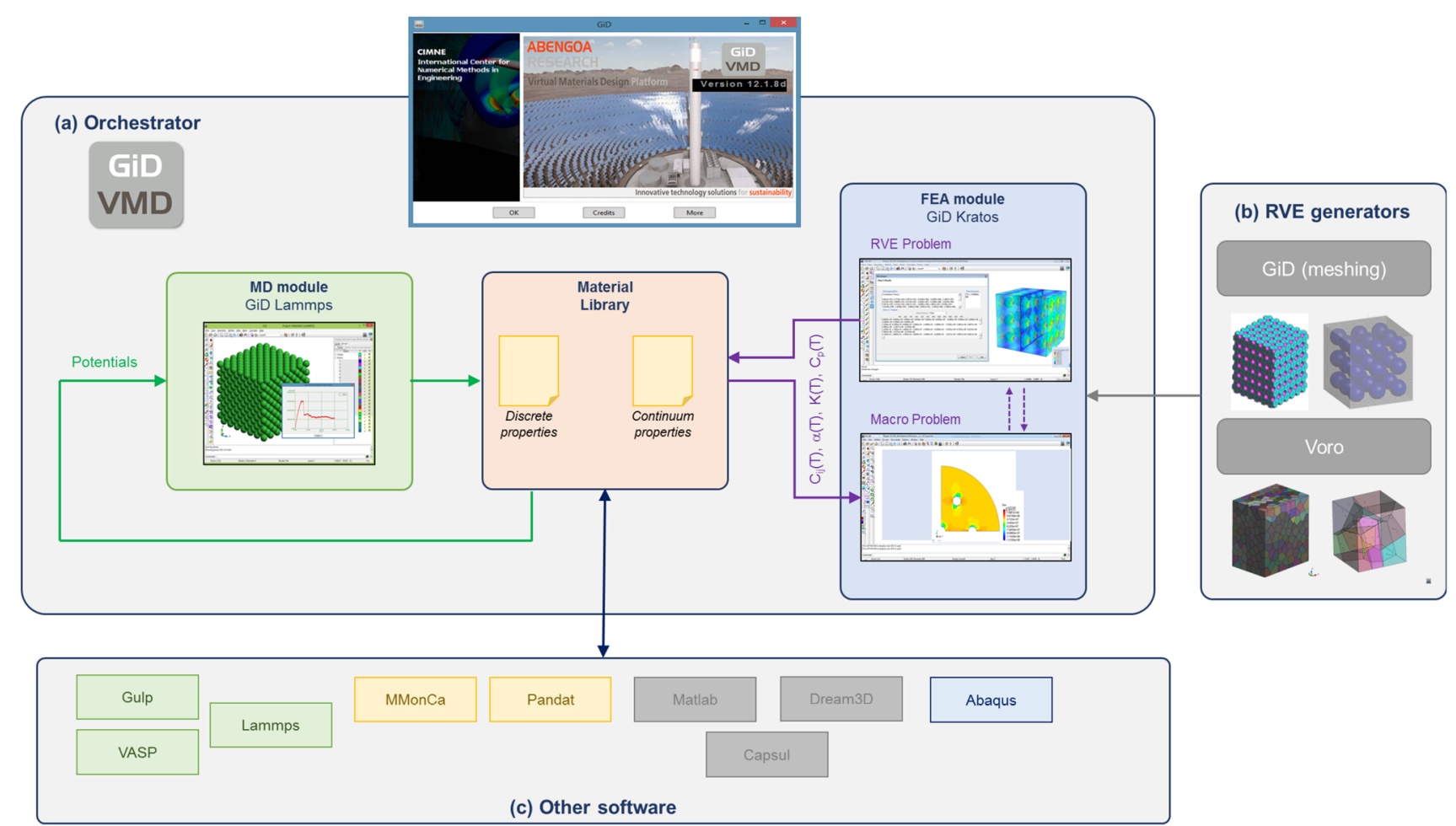

Fig. 4. The VMD project simulation platform: (a) GiD_VMD orchestrator including the MD and FEA modules connected via the material library, (b) RVE generation plugins, and (c) third software used in the project.

additives may enhance the TES performance but also their thermophysical properties $\left(K, C_{\mathrm{p}}, \Delta H_{\mathrm{f}}\right)$ were used at higher levels to characterize the system.

\section{SOFTWARE}

The analysis of the problems described above required the use of different software modules. Indeed, one of the main results of the VMD project was the integration of several modules into a unique simulation platform.

\section{Software Modules Available}

Due to the vast nature of the VMD project, several tools have been used to solve the problems posed at different scales of observation. These are summarized next:

- DFT: VASP, ${ }^{45}$ Gulp. $^{46}$

- MD: Lammps, ${ }^{47}$ GiD-Lammps. ${ }^{48}$

- KMC: MMonCa. ${ }^{49}$

- CTD: Pandat. ${ }^{50}$

- CPFEM: Capsul ${ }^{51}$

- FEA: Kratos Multiphysics ${ }^{52}$ Abaqus FEA. ${ }^{53}$

- RVE generation: GiD, ${ }^{54}$ Voro+++ ${ }^{55}$ Dream3D,${ }^{56}$ Matlab. ${ }^{57}$

\section{GiD-VMD}

The commercial software GiD, developed by CIMNE, was used as the orchestrator of the integrated platform (Fig. 4a). GiD is a universal and user-friendly pre- and postprocessor for numerical simulations. It was used as the interface of the two simulation modules: MD and FEA.

GiD_Lammps is a module that generates the MD scripts for Lammps through a graphical user interface. This module was designed in order to include default preferences such as the potentials used in the reference problems (e.g., EAM, REBO, AIREBO, 2NN-MEAM), problem type (e.g., uniaxial test), or required output data (e.g., elastic constants, CTE, $K, C_{\mathrm{p}}$ ). The output data can be stored in the material library, which can be retrieved from the FEA module.

GiD_Kratos was developed as an interface of Kratos Multiphysics, ${ }^{52}$ and is the FEA tool used to define and solve both the microstructural and macroscopic problems. It accounts for the essential features of these analyses (e.g., cohesive elements, plasticity models, thermal analysis). GiD_Kratos also allows for the definition of a micro- and macrostructure and has $\mathrm{FE}^{2}$ solver capabilities. ${ }^{29}$ Moreover, the module is connected to other $\mathrm{GiD}$ plugins, which were developed on-purpose for this project. Among these, the RVE generators (Fig. 4b) 
are to be highlighted as they can generate Voronoi tessellations or spherical inclusions. This suite of GiD plugins, MD, and FEA modules with unique features was released within this project under the name GiD_VMD, and allowed the interaction with the rest of the tools used (Fig. 4c) through its material library.

\section{CONCLUSION}

Solar thermal energy has become an attractive, sustainable alternative to fossil fuel combustion; however, its full market penetration demands the increase of the operating temperatures and the design of large-scale TES systems. In this work, we have presented the principal outcomes of the Virtual Materials Design Project, an ICME-based approach led by the renewable energy company Abengoa Research during years 2012-2016, whose primary objective was a better understanding of the thermomechanical behavior of critical components in solar thermal power plants.

In this project, six scales of observation were covered, involving more than 30 researchers from four different research groups, and more than ten software modules were extensively used in the resolution of the posed problems. As a result, a complete thermoelastic multiscale analysis, from quantum to continuum mechanics, was carried out for Inconel 625 receiver tubes, and new PCM-based TES devices were analyzed, including the virtual design of new blends of solar salts. One of the main outcomes of this project is the development and integration of a single simulation platform, combining MD and FEA modules, the so-called GiD_VMD platform. From our experience, the decision on following a problem-based approach was successful, not only for solving technical issues but also for the management of a high cross-disciplinary team, composed of chemists, material scientists, and engineers.

Finally, substantial economic benefits may be achieved by following an ICME approach in the energy sector as it certainly reduces the cost of large-scale prototypes, decreasing the development times and maintenance costs due to a better understanding of materials behavior.

\section{ACKNOWLEDGEMENTS}

The authors gratefully acknowledge the funding provided by Abengoa S.A. within the framework of the VMD project. The authors would also like to acknowledge all the researchers that collaborated in this project during the $2012-2016$ period.

\section{REFERENCES}

1. F. Creutzig, P. Agoston, J.C. Goldschmidt, G. Luderer, G. Nemet, and R.C. Pietzcker, Nat. Energy 2, 17140 (2017).

2. C. Parrado, A. Girard, F. Simon, and E. Fuentealba, Energy 94, 422-430 (2016).

3. H. Zhang, W. Kong, T. Tan, and J. Baeyens, Energy 139, 52-64 (2017).
4. National Research Council, Integrated Computational Materials Engineering: A Transformational Discipline for Improved Competitiveness and National Security (Washington, DC: The National Academies Press, 2008).

5. W.A. Curtin, A. Needleman, M. Ortiz, R. Phillips, E. Kaxiras, G. Cedar, and D. Farkas, Virtual Design and Testing of Materials: A Multiscale Approach (Providence, RI: Brown University, 2006).

6. National Nuclear Security Administration, Program Statement for the Advanced Simulation and Computing (ASC) Predictive Science Academic Alliance Program (PSAAP) (2008).

7. J. Allison, D. Backman, and L. Christodoulou, JOM 58, 2527 (2006).

8. M.F. Horstemeyer, Integrated Computational Materials Engineering (ICME) for Metals: Using Multiscale Modeling to Invigorate Engineering Design with Science (New York: Wiley, 2012).

9. J. Llorca and C. González, Virtual mechanical testing of composites: from materials to components, in Proceedings of the 1st World Congress on Integrated Computational Materials Engineering (ICME), The Minerals, Metals \& Materials Society (TMS) (New York: Wiley, 2011), pp. 121127.

10. J. LLorca, C. González, J.M. Molina-Aldareguía, J. Segurado, R. Seltzer, F. Sket, M. Rodríguez, S. Sádaba, R. Muñoz, and L. Canal, Adv. Mater. 23, 5130-5147 (2011).

11. D. Ball, T. Limer, and R. Bridges, A case study on the application of ICME in aircraft design, in 53rd AIAA/ ASME/ASCE/AHS/ASC Structures, Structural Dynamics and Materials Conference, Structures, Structural Dynamics, and Materials and Co-located Conferences (2012).

12. R.J. Glamm, D.M. Rosenbladt, E.D. Pripstein, and J.D. Cotton, Recent progress in implementation of ICME for metallic materials in the airframe industry, in 56th AIAA/ ASCE/AHS/ASC Structures, Structural Dynamics, and Materials Conference, AIAA SciTech Forum, (AIAA 20150199).

13. M. Sangid, J.F. Matlik, A. Keskin, B.H. Thacker, B.J. Bichon, D.L. Ball, S.P. Engelstad, C. Ward, V. Venkatesh, H.A. Kim, V. Saraf, and R. Gorham, Integrating ICME practices into design systems and structural analysis, in 55th AIAA Aerospace Sciences Meeting, AIAA SciTech Forum, (AIAA 2017-0874).

14. J. Gong, D. Snyder, T. Kozmel, C. Kern, J.E. Saal, I. Berglund, J. Sebastian, and G. Olson, JOM 69, 880 (2017).

15. D.G. Backman, D.Y. Wei, D.D. Whitis, M.B. Buczek, P.M. Finnigan, and D. Gao, JOM 58, 36-41 (2006).

16. B. Cowles, D. Backman, and R. Dutton, Integr. Mater. Manuf. Innov. 1, 2 (2012).

17. J. Allison, M. Li, C. Wolverton, and X. Su, JOM 58, 28-35 (2006).

18. V. Savic, L. Hector, H. Ezzat, A. Sachdev, J. Quinn, R. Krupitzer, and X. Sun, SAE Tech Pap, 2015-01-0459 (2015).

19. A. Shaik, Y. Kalariya, R. Pathan, and A. Salvi, ICME based hierarchical design using composite materials for automotive structures, in Proceedings of the 4th World Congress on Integrated Computational Materials Engineering (ICME 2017), The Minerals, Metals \& Materials Series, ed. P. Mason, et al. (Springer, Cham, 2017).

20. W.J. Joost and P.E. Krajewski, Scr. Mater. 128, 107-112 (2017).

21. W.J. Joost, JOM 64, 1032-1038 (2012).

22. H. Xu, Y. Li, and D. Zeng, SAE Int. J. Mater. Manuf. 10, 274-281 (2017).

23. L. Robinson, JOM 63, 30-34 (2011).

24. S.J. Zinkle, K.A. Terrani, and L.L. Snead, Curr. Opin. Solid State Mater. Sci. 20, 401-410 (2016).

25. A. Cruzado, B. Gan, M. Jimenez, D. Barba, K. Ostolaza, A. Linaza, J.M. Molina-Aldareguia, J. Llorca, and J. Segurado, Acta Mater. 98, 242-253 (2015). 
26. Abengoa Research, Abengoa Research Strategic Research Agenda, Abengoa, Seville, Spain, unpublished research (2012).

27. J. Fish, Multiscale Methods: Bridging the Scales in Science and Engineering (Oxford: Oxford University Press, 2010).

28. F. Feyel and J.L. Chaboche, Comput. Methods Appl. Mech. Eng. 183, 309-330 (2000).

29. J. Segurado, R.A. Lebensohn, J. LLorca, and C.N. Tomé, Int. J. Plast 28, 124-140 (2012).

30. F. Montero-Chacón, S. Zaghi, R. Rossi, E. García-Pérez, I. Heras-Pérez, X. Martínez, S. Oller, and M. Doblaré, Finite Elem. Anal. Des. 127, 31-43 (2017).

31. J.M. Ortiz-Roldan, A.R. Ruiz-Salvador, S. Calero, F. Montero-Chacón, E. García-Pérez, J. Segurado, I. Martin-Bragado, and S. Hamad, Phys. Chem. Chem. Phys. 17, 1591215920 (2015).

32. J.M. Ortiz-Roldan, G. Esteban-Manzanares, S. Lucarini, S. Calero, J. Segurado, A.R. Ruiz-Salvador, S. Hamad, and F. Montero-Chacón, Fitting electron density as a physically sound basis for the development of interatomic potentials of complex alloys, unpublished research (2017).

33. W. Chen, G. Xu, I. Martin-Bragado, and Y. Cui, Solid State Sci. 41, 19-24 (2015).

34. ASTM B444-16e1, Standard Specification for NickelChromium-Molybdenum-Columbium Alloys (UNS N06625 and UNS N06852) and Nickel-Chromium-MolybdenumSilicon Alloy (UNS N06219) Pipe and Tube (West Conshohocken, PA: ASTM International, 2016).

35. E. Shapiro and G.E. Dieter, Metall. Trans. 1, 1711-1719 (1970).

36. J.P. Pedron and A. Pineau, Mater. Sci. Eng. 56, 143-156 (1982).

37. C.M. Kuo, Y.T. Yang, H.Y. Bor, C.N. Wei, and C.C. Tai, Mater. Sci. Eng., A 510, 289-294 (2009).

38. M. Prieto-Depedro, I. Martin-Bragado, and J. Segurado, Int. J. Plast 68, 98-110 (2015).

39. L.P. Kadanoff, J. Stat. Phys. 137, 777-797 (2009)

40. H. Ledbetter and R.P. Reed, J. Phys. Chem. Ref. Data 2, 531-618 (1973).
41. G. Martin, N. Ochoa, K. Sa, E. Herv-Luanco, and G. Cailletaud, Int. J. Solids Struct. 51, 1175-1187 (2014).

42. T. Luther and C. Könke, Eng. Fract. Mech. 76, 2332-2343 (2009).

43. H.L. Lukas, S.G. Fries, and B. Sundman, Computational Thermodynamics: The Calphad Method, Vol. 131 (Cambridge: Cambridge University Press, 2007).

44. J.I. Beltrán, J. Wang, F. Montero-Chacón, and Y. Cui, Sol. Energy 155, 154-166 (2017).

45. J. Hafner, J. Comput. Chem. 29, 2044-2078 (2008).

46. J.D. Gale, J. Chem. Soc., Faraday Trans. 93, 629-637 (1997).

47. S. Plimpton, P. Crozier, and A. Thompson, Sandia Natl. Lab. 18, 43 (2007).

48. CIMNE. GiD-LAMMPS: GiD Problem Type for LAMMPS Molecular Dynamics Code, unpublished research (2015).

49. A. Martin-Bragado, G. Rivera, J.L. Valles, and M.J. Gomez-Selles, Caturla. Comput. Phys. Commun. 184, 27032710 (2013)

50. S.L. Chen, S. Daniel, F. Zhang, Y.A. Chang, X.Y. Yan, F.Y. Xie, and W.A. Oates, Calphad 26, 175-188 (2002).

51. IMDEA Materials, Capsul (2015). https://materials.imdea. org/research/simulation-tools/capsul/. Accessed 1 April 2018.

52. P. Dadvand, J. Mora, C. González, A. Arraez, P. Ubach, and E. Oñate. Kratos: an object-oriented environment for development of multi-physics analysis software, in WCCM $V$, Fifth World Congress on Computational Mechanics (2002).

53. Simulia, ABAQUS 6.13 User's Manual (Providence, RI: Dassault Systems, 2013).

54. R. Ribó, M. Pasenau, E. Escolano, J. Pérez, A. Coll, and A. Melendo, GiD The Personal Pre and Postprocessor. Reference Manual, CIMNE, Barcelona, Spain, unpublised research (2006)

55. C. Rycroft, Voro++: a three-dimensional Voronoi cell library in $\mathrm{C}++(2009)$.

56. M.A. Groeber and M.A. Jackson, Integr. Mater. Manuf. Innov. 3, 5 (2014).

57. MATLAB 2014b (The Mathworks, Inc., Natick, MA). 